EPJ Web of Conferences 35, 06005 (2012)

DOI: $10.1051 /$ epjconf/20123506005

(C) Owned by the authors, published by EDP Sciences, 2012

\title{
Free-ion hyperfine fields and magnetic-moment measurements on radioactive beams
}

\author{
Andrew E. Stuchbery ${ }^{1, a}$ \\ Department of Nuclear Physics, Australian National University, Canberra ACT 0200, Australia
}

\begin{abstract}
The recoil in vacuum (RIV) technique for magnetic-moment measurements on radioactive beams is discussed with examples from the ${ }^{132} \mathrm{Sn}$ region. Attention then turns to recoil in vacuum and the free-ion hyperfine fields of Se and Ge ions leaving carbon foils with velocities of about $5 \%$ of the speed of light. These notionally similar ions show unexpected differences which must stem from differences in their atomic structure.
\end{abstract}

\section{Introduction: radioactive beams and nuclear moments}

New scientific opportunities have been opened up by the availability of beams of rare isotopes produced by radioactive beam accelerators. This frontier of nuclear physics research will continue for some decades as new facilities come on line internationally. The research program at the Heavy Ion Accelerator Facility at the Australian National University seeks to engage with this frontier in several ways. The approach discussed in this paper is to use the Australian facility to develop experimental methods and perform experiments on stable beams that underpin experiments on the international radioactive beam facilities. Our work on excited-state magnetic moment measurements has successfully followed this path over recent years [1-4].

The magnetic moment, which is measured by observing the response of the nuclear spin to an applied magnetic field, is an important observable in the study of the quantum mechanics of nuclear excitations. For example, it gives insights into how the nucleus carries its angular momentum. Magnetic moments are very sensitive to singleparticle aspects of the wavefunction, and can strongly distinguish between proton versus neutron excitations.

Our radioactive beam studies have focused on the firstexcited $2^{+}$states of neutron-rich nuclei near closed shells. These states typically have lifetimes of the order of picoseconds, thus requiring the use of intense hyperfine fields to perturb the orientation of the nuclear state by a measurable amount during its brief lifetime. This paper discusses the recoil in vacuum (RIV) method, which makes use of the free-ion hyperfine fields of highly charged ions recoiling out of a target into vacuum.

Experiments with radioactive beams are challenging in a number of ways. Firstly, the beam intensity is low, often orders of magnitude weaker than stable beams. To compensate requires the use of sophisticated high-efficiency detector arrays. Secondly, radioactive beams can be contaminated with unwanted isobaric ions. This complication must be evaluated case-by-case. Thirdly, because the beams are radioactive any experiment which aims to study the beam ion itself (rather than its decay products) must en-

\footnotetext{
a e-mail: andrew.stuchbery@anu.edu.au
}

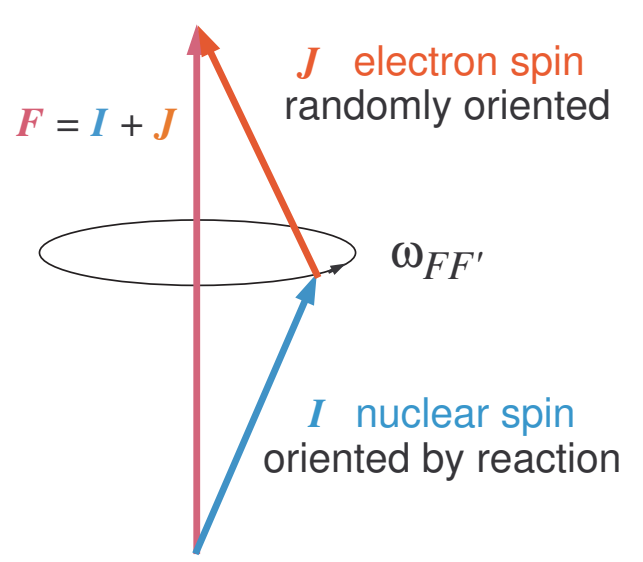

Fig. 1. Vector model of the free-ion hyperfine interaction.

sure that the beam does not stop in view of the $\gamma$-ray detectors. Finally, radioactive beam experiments almost all use 'inverse kinematics' wherein the roles of beam and target are interchanged. For example, a rare isotope radioactive beam of interest may be Coulomb excited on a stable target to discover excited states, and/or to measure reduced transition probabilities and magnetic moments.

\section{The RIV method and its application to radioactive beams}

When a free ion moves through vacuum the hyperfine interaction couples the atomic spin $\boldsymbol{J}$ to the nuclear spin $\boldsymbol{I}$ and together they precess about the total spin $\boldsymbol{F}=\boldsymbol{I}+\boldsymbol{J}$, as illustrated in Fig. 1. The precession frequency $\omega_{F F^{\prime}}$ is proportional to the nuclear $g$ factor and the magnitude of the hyperfine magnetic field at the nucleus. To measure the $g$ factor, the nuclear state of interest is excited by a suitable reaction and then allowed to recoil into vacuum. The effect of the hyperfine interaction is observed via the perturbation of the angular correlation of the $\gamma$-rays de-exciting the state. The concept is illustrated in Fig. 2 using data from the Holifield Radioactive Ion Beam Facility (HRIBF) at Oak Ridge National Laboratory [1]. 
UNPERTURBED
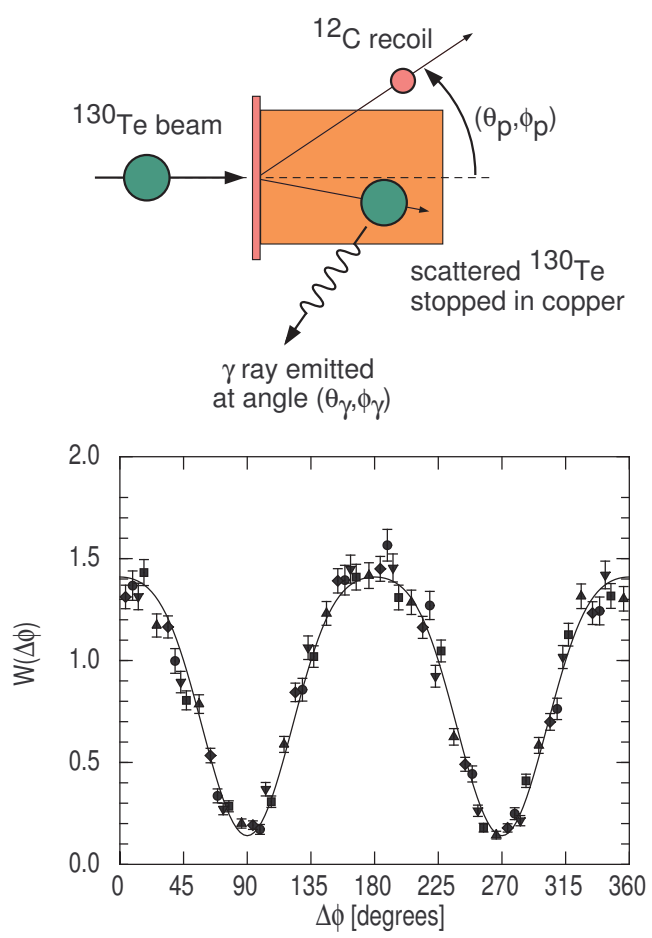

PERTURBED
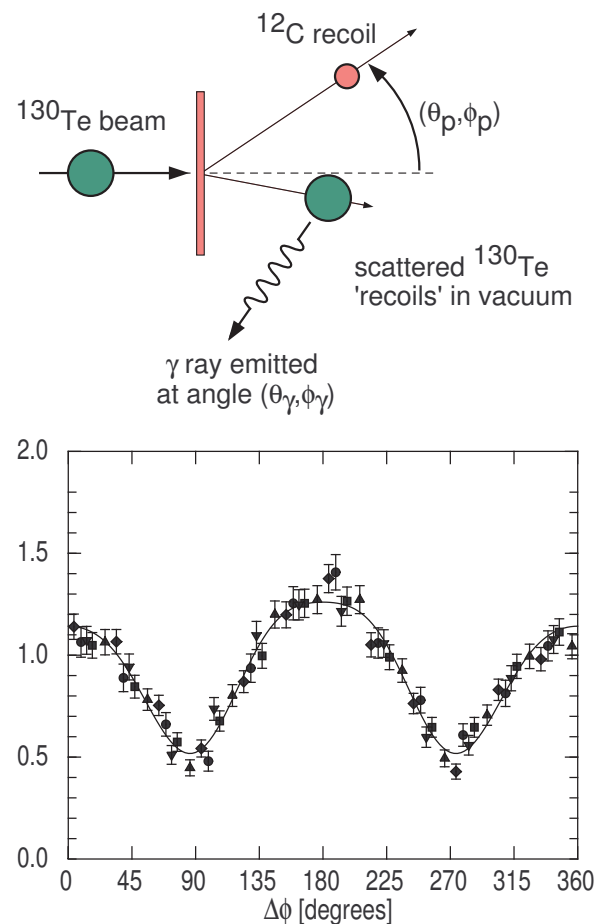

Fig. 2. Unperturbed and perturbed angular correlations for the $2_{1}^{+} \rightarrow 0_{1}^{+}$transition in ${ }^{130} \mathrm{Te}$ [1]. Left: Unperturbed angular correlations following implantation into copper. Right: Perturbed angular correlations with reduced anisotropy resulting from vacuum deorientation of the nuclear spin.

In the presence of vacuum deorientation, the particle$\gamma$ angular correlation after Coulomb excitation takes the form (see e.g. Ref. [5] and references therein)

$$
W\left(\theta_{p}, \theta_{\gamma}, \Delta \phi\right)=\sum_{k q} B_{k q}\left(\theta_{p}\right) Q_{k} G_{k} F_{k} D_{q 0}^{k *}\left(\Delta \phi, \theta_{\gamma}, 0\right),
$$

where the angles are defined schematically in Fig. 2 and $\Delta \phi=\phi_{p}-\phi_{\gamma}$. The attenuation coefficients, $G_{k}$, specify the vacuum deorientation effect; $B_{k q}\left(\theta_{p}\right)$ is the statistical tensor, which defines the spin alignment of the initial state. $F_{k}$ represents the usual $F$-coefficient for the $\gamma$-ray transition, $Q_{k}$ is the attenuation factor for the finite size of the $\gamma$-ray detector, and $D_{q 0}^{k *}\left(\Delta \phi, \theta_{\gamma}, 0\right)$ is the rotation matrix. In the applications of interest $k=0,2,4$.

The time-dependent attenuation coefficient for an electronic configuration of spin $J$, which produces a magnetic field $B$ at the nucleus, and with nuclear $g$ factor, $g$, is given by [6]

$$
G_{k}(t)=\sum_{F, F^{\prime}} \frac{(2 F+1)\left(2 F^{\prime}+1\right)}{2 J+1}\left\{\begin{array}{ccc}
F & F^{\prime} & k \\
I & I & J
\end{array}\right\}^{2} \cos \left(\omega_{F F^{\prime}} t\right),
$$

where

$$
\omega_{F F^{\prime}}=g \frac{\mu_{\mathrm{N}}}{\hbar} B \frac{\left(F(F+1)-F^{\prime}\left(F^{\prime}+1\right)\right)}{2 J} .
$$

The experiments considered here determine the time-integral attenuation factors

$$
G_{k}(\infty)=\int_{0}^{\infty} G_{k}(t) \mathrm{e}^{-t / \tau} \mathrm{d} t / \tau
$$

where $\tau$ is the mean life of the nuclear state.

For an ensemble of many-electron ions with a distribution of electron configurations the attenuation coefficient is given by

$$
G_{k}=\sum_{i} w_{i} G_{k}^{i},
$$

where $G_{k}^{i}$ is the deorientation coefficient for an ion in the state $i$ and $w_{i}$ is the fraction of ions in that state. The weights are normalized so that $\sum w_{i}=1$.

A superposition of many hyperfine frequencies gives a quasi-exponential time dependence to the vacuum attenuation factors, $G_{k}(t)$. Thus the alignment of the nuclear state, and hence the anisotropy of the $\gamma$-ray angular correlation, decreases approximately exponentially with time, at a rate that depends on the magnitude of the nuclear $g$ factor. In the case where $w_{i}$ is a Lorentzian distribution centered at $B=0$, the time-integrated attenuation coefficient is given by

$$
G_{k}(\infty)=\alpha_{k}+\left(1-\alpha_{k}\right) \frac{1}{1+\left|\Gamma_{k}\right| \tau},
$$

where $\Gamma_{k}$ is proportional to $|g|$. The 'hard core' parameter $\alpha_{k}$ gives the asymptotic value of $G_{k}$ at long times, whereas $\Gamma_{k}$ is the time constant for the quasi-exponential decay of the attenuation coefficient. Physically, $\Gamma_{k} / g$ is related to the average strength of the hyperfine fields acting on the nucleus, while $\alpha_{k}$ is determined by the average angular momentum of the atomic electron configurations. Although a Lorentzian distribution of hyperfine fields is not realistic, Eq. (6) has proved useful for fitting data. 


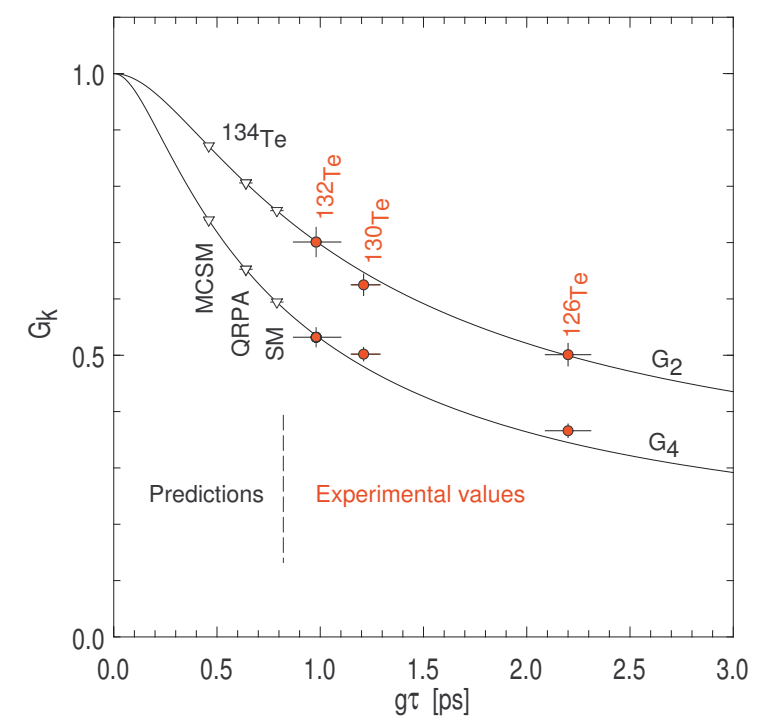

Fig. 3. Attenuation factors versus $g \tau$ for several isotopes of Te. For semimagic ${ }^{134} \mathrm{Te}$ the three points correspond to predicted $g$ factors: Monte Carlo Shell Model (MCSM) [8], Quasiparticle Random Phase Approximation (QRPA) [9], Shell Model (SM) [10].

Figure 3 illustrates the procedure used to determine the $g\left(2_{1}^{+}\right)$value in neutron-rich ${ }^{132} \mathrm{Te}$ based on a calibration using stable ${ }^{126} \mathrm{Te}$ and ${ }^{130} \mathrm{Te}$. The measured attenuation factors are plotted versus the product $g \tau$. The $g$ factors for the stable isotopes were measured precisely at the Australian National University [7]. The curves represent a model-based fit to the calibration data [4]. Fig. 3 also shows the locations of points on the $G_{k}$ versus $g \tau$ curve as predicted by various nuclear models for $g\left(2_{1}^{+}\right)$in ${ }^{134} \mathrm{Te}$ [810]. It is evident that the range of predicted $G_{k}$ values for semimagic ${ }^{134} \mathrm{Te}$ is near the maximum slope of the $G_{k}$ vs $g \tau$ curve, and hence the RIV measurement has the sensitivity required to distinguish between the theoretical models. In the coming months we expect to complete the analysis of an RIV measurement on ${ }^{134} \mathrm{Te}$ performed at HRIBF in February 2012 [11].

\section{Characterizing Free ion fields}

The $G_{k}$ versus $g \tau$ curve must be accurately characterized to extract $g$ factors from RIV measurements. To this end, at the Australian National University we have been studying the free-ion hyperfine fields of stable nuclei having known moments. Like the radioactive beam measurements, the stable-beam studies have been performed in inverse kinematics with the beam ions Coulomb excited on either ${ }^{12} \mathrm{C}$ or ${ }^{27} \mathrm{Al}$ targets; the different targets serve to vary the exit velocity and hence charge-states of the ions entering vacuum.

Figure 4 shows measured attenuation coefficients for $175 \mathrm{MeV}$ beams of the stable ${ }_{32} \mathrm{Ge}$ and ${ }_{34} \mathrm{Se}$ isotopes excited on a carbon target. The $g$ factors of these isotopes are taken from Mertzimekis et al. [12], and the lifetimes from Raman et al. [13]. The observed difference in the attenuation factors for the longer-lived isotopes, i.e. ${ }^{74,76} \mathrm{Ge}$ compared with ${ }^{76,82} \mathrm{Se}$, was surprising. We do not yet have a 'first-principles' atomic physics explanation for this difference. However some important conclusions can be drawn based on empirical and semi-empirical fits to the data. The left panel in Fig. 4 shows an empirical fit based on Eq. (6); the right panel shows a semi-empirical model-based fit. This static-model fit method has been described in [4]. It assumes that the vacuum attenuation results from the superposition of hyperfine fields with Gaussian distributions in atomic spin and field strength, cf. Eq. (5). To limit the parameters it has proved effective to (i) fix the standard deviation of the atomic spin distribution to $\sigma_{J}=1 \hbar$, and (ii) for the distribution of hyperfine fields, to set $\sigma_{B}=\bar{B}$, where $\bar{B}$ is the average hyperfine field strength at the nucleus. Two parameters, $\bar{J}$ and $\bar{B}$, then determine $G_{2}$ and $G_{4}$.

Both the empirical fit and the semi-empirical modelbased fit show that effectively the same average hyperfine field strength is experienced by the Ge and Se ions. What differs for the Ge versus Se ions is the magnitude of the hard core term in the empirical fit or, equivalently, the value of $\bar{J}$ in the model-based fit. Specifically, the modelbased fit gives $\bar{J}=1.2$ for Ge and $\bar{J}=1.7$ for Se.

Charge-state measurements indicate that the Ge ions here are mainly $\mathrm{Mg}$-like and Al-like, whereas the Se ions are predominantly P-like. As a first step towards a 'microscopic' model of the RIV hyperfine interactions for these ions, the hyperfine fields at the nucleus were evaluated for Mg-like Ge ions using the multiconfiguration HartreeFock (MCHF) Atomic Structure Package [14,15]. Multiconfiguration calculations were performed to evaluate (at least approximately) the effects of core polarization. The lowest 35 levels of Ge XXI from the configurations $3 s^{2}$, $3 s 3 p, 3 p^{2}, 3 s 3 d$, and $3 d^{2}$ were considered. These atomic levels are estimated to have lifetimes of the order of the nuclear lifetimes (up to 20) ps or longer [16]. Higher atomic states tend to be shorter lived than the nuclear lifetimes and therefore will have less influence. The calculated hyperfine fields are shown in the left panel of Fig. 5. Of note are the ${ }^{3} P_{J}(J=0,1,2)$ levels from the $3 s 3 p$ configuration, which form the lowest excited states of the ion, produce the strongest hyperfine fields $(J=1,2)$, and have lifetimes that are orders of magnitude longer than the nuclear lifetimes. It is likely that these atomic states are important in determining the hard-core values of the attenuation coefficients.

A simple microscopic approach to model the RIV attenuation for $\mathrm{Mg}$-like $\mathrm{Ge}$ ions would be to superimpose the deorientation coefficients for the calculated hyperfine fields up to a cut-off in excitation energy, assuming a weighting factor of $(2 J+1)$ for each atomic state. Because Al-like ions are about as important in the experiments as Mg-like ions, a comparison with the experimental data can only be qualitative. Nevertheless, it is useful to note that to reproduce the behavior of the observed attenuations (and hence the average hyperfine field strength) requires a cut-off at about $150 \mathrm{eV}$ in the excitation energy of $\mathrm{Mg}$-like $\mathrm{Ge}$ ions. The right panel of Fig. 5 compares the distribution of hyperfine fields for the first 35 states of $\mathrm{Mg}$-like Ge ions with the distribution used in the model-based fit to the experimental data in Fig. 4. It is evident that the calculated hyperfine fields have the required strength to explain the data, and that excluding states above $150 \mathrm{eV}$ in excitation energy brings the distribution of fields closer to the empirically determined Gaussian distribution. 


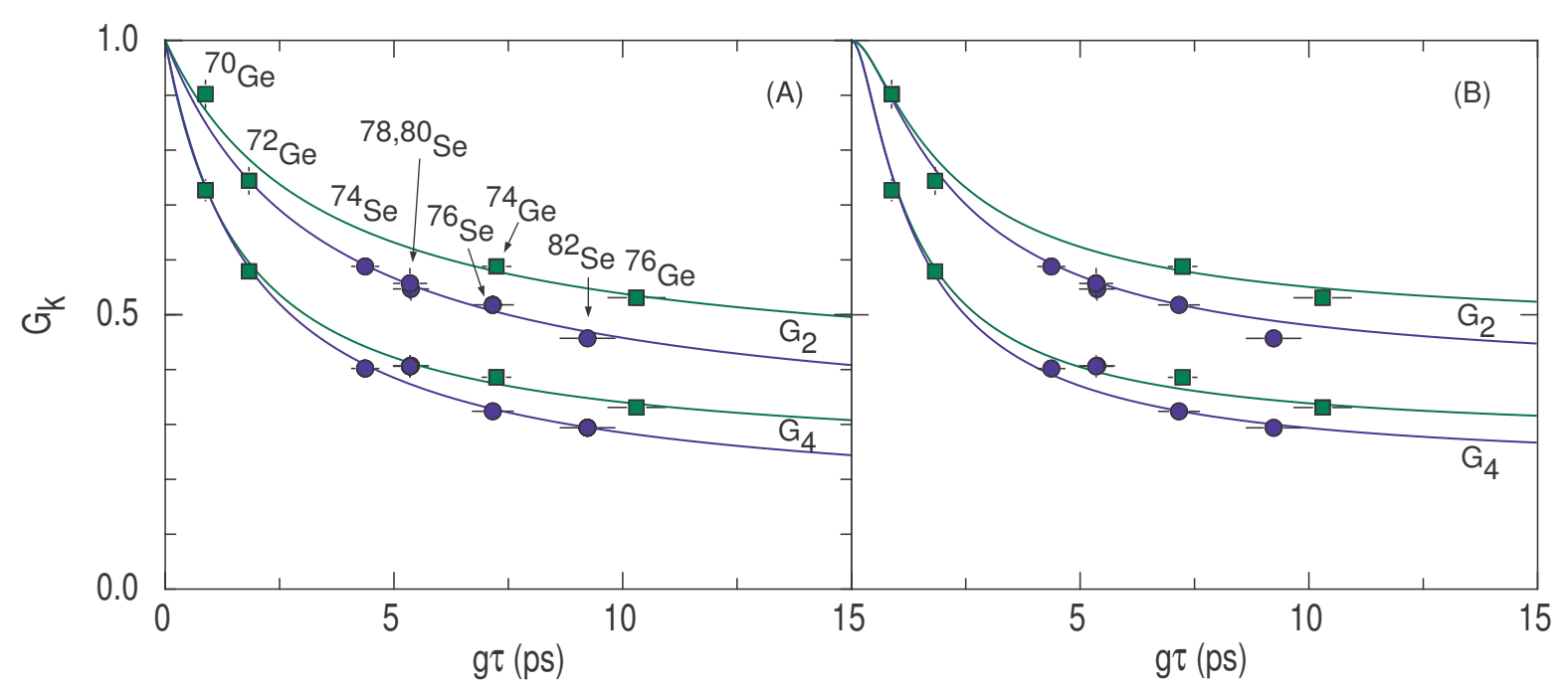

Fig. 4. Vacuum deorientation coefficients for the stable isotopes of $\mathrm{Ge}$ and $\mathrm{Se}$ after excitation of $175 \mathrm{MeV}$ beams on carbon targets. (A) Solid lines are empirical fits to equation 6. (B) Lines are model-based fits assuming Gaussian field distributions.
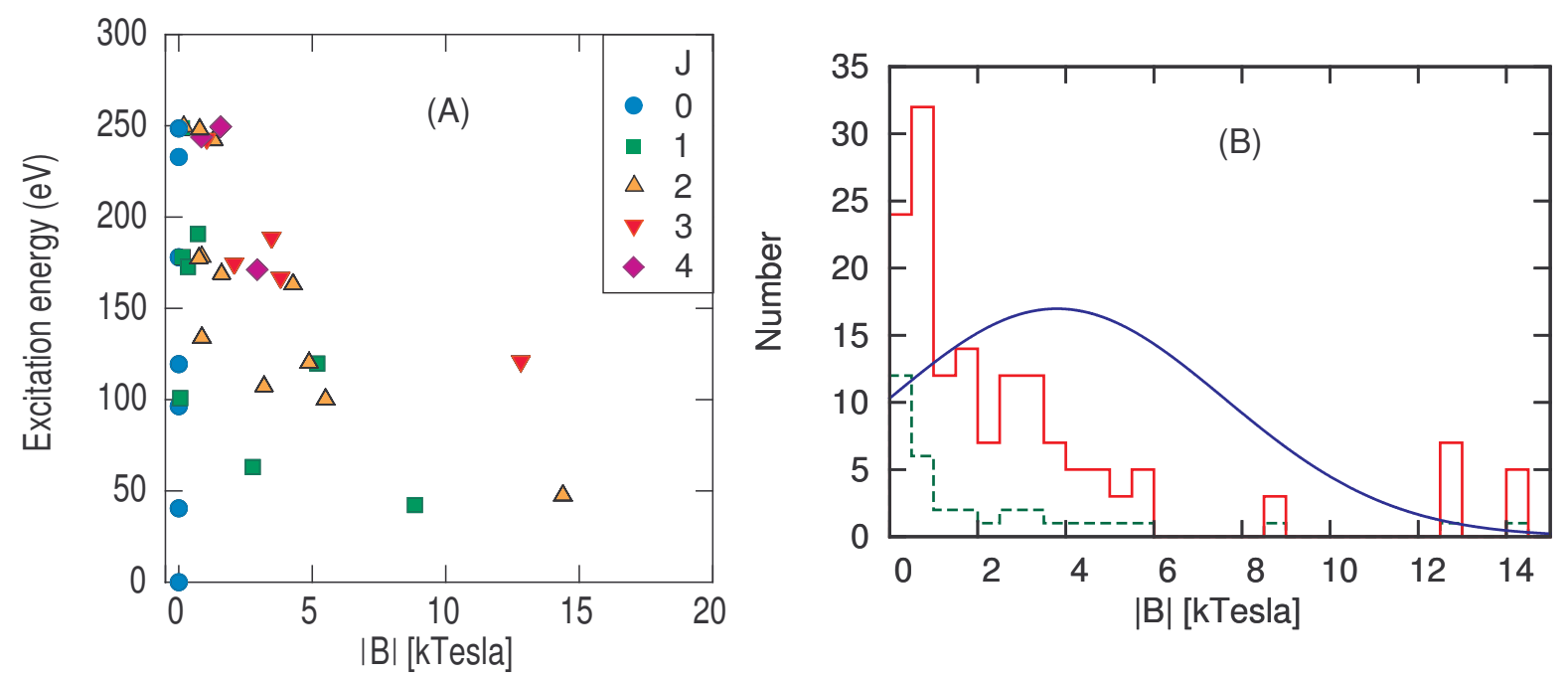

Fig. 5. (A) Experimental excitation energies versus hyperfine magnetic field strengths for Mg-like Ge ions. The symbols and colors identify the angular momentum of each state. (B) Histogram of hyperfine field strengths for Mg-like Ge (dashed green histogram) from the configurations $3 s^{2}, 3 s 3 p, 3 p^{2}, 3 s 3 d$ and $3 d^{2}$. The same field distribution with a statistical $(2 J+1)$ factor is shown as the solid red histogram. The blue curve indicates the shape of the Gaussian field distribution which fits the data in Fig. 4.

The next steps for more realistic modeling are: first, to include the range of ionic charge states contributing to the observed attenuation coefficients, and second, to make quantitative estimates of the atomic decays taking place on the order of the nuclear lifetime. Taking into account the atomic lifetimes will reduce the impact of higher-excited low-field states on the observed RIV attenuation. Once these well defined processes are taken into account we can evaluate whether the initial population distribution of the atomic states of the ions, as they exit from the target and enter vacuum, has a significant impact on the observed attenuation coefficients.

To sum up, atomic structure effects are seen in otherwise similar measurements of free-ion hyperfine fields for Ge and Se ions carrying $\sim 12-15$ electrons. The difference stems from a difference in the average atomic angular momentum in the range between $\bar{J}=1$ and $\bar{J}=2$, an aspect that is discussed further in [17]. Atomic calculations have commenced with the goal to develop a quantitative microscopic model of the free-ion hyperfine fields. The aim of the present work is to underpin magnetic moment measurements on radioactive beams at international facilities. In future work, the experience with the atomic structure calculations for free ions will be adapted and applied to calculations of Auger spectra for medical isotopes, as described elsewhere $[18,19]$.

\section{Acknowledgments}

The author is grateful to many colleagues who have made the work discussed here possible. Russell Leslie is acknowledged for his contributions to the measurements described in section 3, and Tibor Kibédi is thanked for discussions 
and assistance with computation. This research was supported in part by the Australian Research Council Discovery Scheme, Grant No. DP0773273.

\section{References}

1. N. J. Stone, et al., Phys. Rev. Lett. 94, (2005) 192501

2. A. D. Davies, et al., Phys. Rev. Lett. 96, (2006) 112503

3. A. E. Stuchbery, et al., Phys. Rev. C. 74, (2006) 054307

4. A. E. Stuchbery, et al., Phys. Rev. C. 76, (2007) 034307

5. A. E. Stuchbery, Nucl. Phys. A 723, (2003) 69

6. G. Goldring, in R. Bock Heavy Ion Collisions Vol. 3, (North-Holland, Amsterdam, 1982) 484

7. A. E. Stuchbery, et al., Phys. Rev. C. 76, (2007) 034306

8. N. Shimizu, et al., Phys. Rev. C 70, (2004) 054313

9. J. Terasaki, et al., Phys. Rev. C 66, (2002) 054313

10. B. A. Brown, et al., Phys. Rev. C 71, (2005) 044317

11. A. E. Stuchbery, J. M. Allmond, A. Galindo-Uribarri, E. Padilla-Rodal, D. C. Radford, J. C. Batchelder, J. R. Beene, N. Benczer-Koller, C. R. Bingham, M. E. Howard, G. J. Kumbartzki, J. F. Liang, B. Manning, S. D. Pain, N. J. Stone, R. L. Varner, C.-H. Yu, to be published

12. T. J. Mertzimekis, A. E. Stuchbery, N. Benczer-Koller, M. J. Taylor, Phys. Rev. C 68, (2003) 054304

13. S. Raman, C. W. Nestor, Jr., P. Tikkanen, Atom. Data Nucl. Data Tables 78, (2001) 1

14. C. Froese Fischer, Comput. Phys. Comm. 128, (2000) 635

15. C. Froese Fischer, T. Brage, and P. Jönsson, Computational Atomic Structure: An MCHF Approach (Institute of Physics, Bristol, 1997)

16. C. Froese Fischer, G. Tachiev, and A. Irimia, Atom. Data Nucl. Data Tables 92, (2006) 607

17. A. E. Stuchbery, Proceedings XXXV Symposium on Nuclear Physics, Cocoyoc, Mexico, January 2012, IOP Conference Series, to be published

18. B. Q. Lee, T. Kibédi, A. E. Stuchbery, and K. A. Robertson, these proceedings

19. B. Q. Lee, T. Kibédi, A. E. Stuchbery, and K. A. Robertson, Computational and Mathematical Methods in Medicine, in press. 